\title{
BMJ Open Implementing communication and decision-making interventions directed at goals of care: a theory-led scoping review
}

\author{
Amanda Cummings, ${ }^{1,2}$ Susi Lund, ${ }^{1,2}$ Natasha Campling, ${ }^{1,2}$ Carl R May, ${ }^{1,2,3}$ \\ Alison Richardson, ${ }^{1,2,3}$ Michelle Myall ${ }^{1,2}$
}

To cite: Cummings A, Lund S, Campling N, et al. Implementing communication and decisionmaking interventions directed at goals of care: a theory-led scoping review. BMJ Open 2017;7:e017056. doi:10.1136/ bmjopen-2017-017056

- Prepublication history and additional material for this paper are available online. To view please visit the journal (http:// dx.doi.org/10.1136/bmjopen2017-017056).

Received 4 April 2017

Revised 10 August 2017

Accepted 11 August 2017

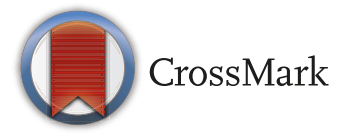

${ }^{1}$ Faculty of Health Sciences, University of Southampton, Southampton, UK

${ }^{2}$ NIHR CLAHRC Wessex, University of Southampton, Southampton, UK

${ }^{3}$ University Hospital Southampton, NHS Foundation Trust, Southampton, UK

Correspondence to Dr Amanda Cummings; ac2a13@soton.ac.uk

\section{ABSTRACT}

Objectives To identify the factors that promote and inhibit the implementation of interventions that improve communication and decision-making directed at goals of care in the event of acute clinical deterioration.

Design and methods A scoping review was undertaken based on the methodological framework of Arksey and 0'Malley for conducting this type of review. Searches were carried out in Medline and Cumulative Index to Nursing and Allied Health Literature (CINAHL) to identify peerreviewed papers and in Google to identify grey literature. Searches were limited to those published in the English language from 2000 onwards. Inclusion and exclusion criteria were applied, and only papers that had a specific focus on implementation in practice were selected. Data extracted were treated as qualitative and subjected to directed content analysis. A theory-informed coding framework using Normalisation Process Theory (NPT) was applied to characterise and explain implementation processes.

Results Searches identified 2619 citations, 43 of which met the inclusion criteria. Analysis generated six themes fundamental to successful implementation of goals of care interventions: (1) input into development; (2) key clinical proponents; (3) training and education; (4) intervention workability and functionality; (5) setting and context; and (6) perceived value and appraisal.

Conclusions A broad and diverse literature focusing on implementation of goals of care interventions was identified. Our review recognised these interventions as both complex and contentious in nature, making their incorporation into routine clinical practice dependent on a number of factors. Implementing such interventions presents challenges at individual, organisational and systems levels, which make them difficult to introduce and embed. We have identified a series of factors that influence successful implementation and our analysis has distilled key learning points, conceptualised as a set of propositions, we consider relevant to implementing other complex and contentious interventions.

\section{INTRODUCTION}

In the event of a patient becoming acutely unwell, treatment and care decisions are recommended by clinicians. While these

\section{Strengths and limitations of this study}

- This paper outlines a scoping review of a broad and diverse literature, both published and grey, focusing on the implementation of goals of care interventions for patients facing clinical deterioration. However, because of its focus on implementation, not all examples of goals of care interventions could be included.

- Normalisation Process Theory (NPT) was used to investigate and explain the successful implementation of interventions, and a theoretical approach has been applied to all stages of the review process.

- In a number of included papers, information relating to implementation barriers was missing, and there was bias towards the presentation of positive outcomes. This may reflect a reluctance to focus on challenges with study authors keen to exhibit and promote the benefits of interventions.

- The review led to goals of care interventions being defined as 'contentious' with a moral purpose and value, and identified the elements and learnings that could be transferable to other examples of such interventions.

We have proposed that contentious interventions consist of components at three levels. Across the literature reviewed, the focus was on components at the individual level where negotiated decisionmaking between participants occurs. There was limited focus on the components that take place within and across organisations and the influence of system constraints. This has important limitations for our interpretation of data and analysis.

decisions are based on the clinical judgement of a healthcare professional, they should also be bound by the preferences and wishes of the patient and their family. Processes and tools (referred to here as goals of care interventions), that provide a framework for discussing and documenting appropriate treatment options in the event of acute clinical deterioration are paramount. 
These interventions aim to improve patient and family involvement, enabling exploration and understanding of the current clinical situation and facilitation of communication and negotiated decision-making about future treatment options. ${ }^{12}$ They offer a means for patients' preferences to be taken into account, improving communication and clarity across the wider clinical team.

We refer to goals of care in the event of acute deterioration, where different levels of treatment might be appropriate and range from full escalation in a critical care environment to symptom control measures. ${ }^{3}$ Goals of care are currently referred to using various terms, including but not confined to ceilings of care, treatment escalation plans and treatment limitations. They exist in numerous formations including a specific, dedicated paper form, a narrative entry in a paper medical record and inclusion in an electronic patient record, and may be introduced as an extension of the 'do not attempt cardio-pulmonary resuscitation' (DNACPR) process. These interventions offer a system for recording recommended treatment and care, ideally including all components of the decision pathway and can be applied across different care settings. They require a process to be created and implemented that is recognised across organisations, takes account of sociolegal frameworks, such as the UK Mental Capacity Act (MCA) (2005), ${ }^{4}$ and is designed to protect individuals who do not have capacity to make decisions about their care and treatment.

Goals of care interventions are complex and consist of multiple interacting components. The number and difficulty of behaviours required by those delivering or receiving such an intervention, variability of outcomes, and degree of flexibility and tailoring that is permitted, all contribute to making this a complex intervention. ${ }^{5}$ As well as being complex, goals of care interventions are established as interactions and recording systems with a moral purpose and value. This means they may be contentious in practice because they contain elements that seek to routinise highly complex clinical skills, practice and different types of wisdom, in a context of uncertainty. Contentiousness can arise because the intervention relies on patient, family and clinician interactions, interclinician interactions (potentially across clinical settings and organisational boundaries), and societal and legal frameworks, such as the MCA (2005) and the European Convention on Human Rights (2002). ${ }^{46}$

In spite of a growing body of literature describing the introduction and benefits of goals of care interventions, little is known about the factors that influence their successful implementation in clinical practice. Here implementation is defined as any deliberately initiated attempt to introduce new, or modify existing, patterns of action in health care or some other formal organizational setting. Deliberate initiation means that an intervention is: institutionally sanctioned; formally defined; consciously planned; and intended to lead to a changed outcome'. 7 As we have previously argued, "this is more than the adoption or diffusion of innovations" as effective implementation is about interventions being made workable and embedded in routine clinical practice. $^{7}$

Understanding and evaluating the implementation of complex interventions in practice remain a challenge for healthcare managers, policy makers and for those who enact them outside of formal research settings. ${ }^{8}$ Furthermore, as interventions found to be effective in the context of health services research studies can fail to translate into meaningful healthcare outcomes across varying contexts, ${ }^{9}$ this makes understanding the reasons for failure or partial success even more essential. Implementation science, which promotes the integration of research findings and evidence into healthcare policy and practice, is increasingly recognised within health services research to make a key contribution to such knowledge. Comprehensive process evaluation of the implementation of healthcare interventions is increasingly important for future learning, ${ }^{10}$ as it enables understanding of transition from closed systems of highly structured research or service development projects into the real world of open systems healthcare delivery where they are operationalised. ${ }^{11}{ }^{12}$ Learning from the available existing knowledge in this area can be used to inform healthcare practice change and contribute to the field of implementation science.

Having previously applied Normalisation Process Theory (NPT) to aid learning in the comparable context of advance care plans, ${ }^{13}$ we have used NPT to characterise and explain implementation processes. ${ }^{14}$ NPT provides a set of tools to investigate and understand the processes through which interventions are operationalised in healthcare settings and incorporated into everyday practice. $^{14-17}$

In this paper, we present a scoping review of goals of care interventions which aims to identify the factors that promote and inhibit the implementation of interventions which improve communication and decision-making directed at goals of care. Using goals of care interventions as an example, a secondary aim of this review is to characterise the components and consider the implications for implementation of, contentious interventions.

\section{DESIGN AND METHODS}

A scoping review was the most appropriate methodology, given the need to extract and map principles from a diverse and broad body of evidence. ${ }^{18} 19$

We used Arksey and O'Malley's five-stage framework for conducting scoping reviews, which includes identifying the research question, identifying relevant literature, selection, charting the data and collating, summarising and reporting the results. ${ }^{20}$ This guided the scoping review and where necessary we developed more specific procedures to inform the review process. Levac et $a l \mathrm{~s}^{21}$ recommendations for refining the methodological application were also incorporated to increase rigour of the review process. 
Table 1 Inclusion and exclusion eligibility criteria

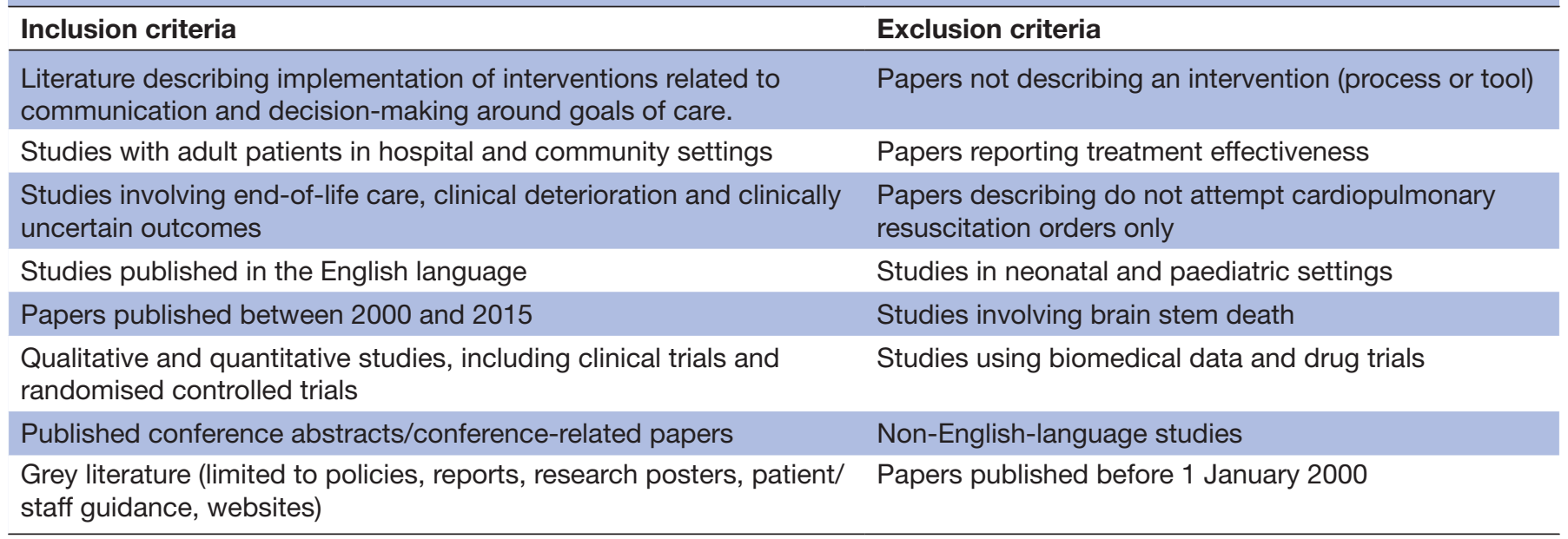

\section{Inclusion and exclusion criteria}

Literature was selected using specific inclusion and exclusion criteria (table 1), and was included if it was considered to have a specific focus on implementation in practice. To identify all relevant literature on goals of care, grey literature was included and actively sought as part of the search strategy. Only papers published from the year 2000 onwards were included as those published earlier were unlikely to reflect current practice. Papers focusing solely on DNACPR orders were excluded due to their focus on only one decisional element of goals of care. Existing evidence also suggests that DNACPR decisions are not always discussed with patients or families. ${ }^{22}$ The combining of DNACPR decisions within wider goals of care interventions has been shown to improve clarity and communication, and the focus of this review is on implementing a process whereby goals of care are discussed. ${ }^{23}$

\section{Search strategy and information sources}

The search strategy was designed to identify primary studies and other literature, both published and unpublished, that met the eligibility criteria. Separate searches were undertaken for primary and grey literature.

\section{Primary literature searches}

The primary literature search was carried out in two stages. The first stage involved an initial search of the bibliographical database Medline using a preliminary keyword search based on the terms of the topic, the text terms used in titles and abstracts, and index terms used to describe articles. Full details of the primary literature search strategy are outlined in online supplementary appendix 1 .

Terms identified in the titles and abstracts of relevant articles produced from stage 1 were used to develop further keywords for the second stage of the literature search (see online supplementary appendix 1). Dual combined keyword searches were conducted in Medline and Cumulative Index to Nursing and Allied Health Literature (CINAHL) bibliographical databases, with a third keyword added if searches produced $>200$ results. In addition, further literature was identified through existing knowledge and networks.

\section{Grey literature searches}

Google searches were conducted using terminology relating to known goals of care interventions identified in an earlier scoping exercise which mapped the use of forms for recording these decisions in the UK. The first 10 results were screened for relevance and further review. A further Google search focused on policies and guidance related to goals of care was undertaken and the first 50 results screened for relevance.

All screened, de-duplicated citations were imported into the bibliographical software management package EndNote. Searches were completed by August 2015. This was due to the imminence of a national programme of work which has led to the Recommended Summary Plan for Emergency Care and Treatment (RESPECT). ${ }^{24}$ Communication and decision-making around goals of care is a growing area of interest, and it is hoped that findings from this review can be used to inform the implementation of such a major advancement in the field.

\section{Screening}

An extensive screening process was undertaken. At the first stage of screening, articles were assessed by two independent reviewers (AC, CRM) based on the information provided in the title. Primary literature judged to be relevant after first screen, and which met the eligibility criteria, were obtained in full text. Articles for which there was disagreement between reviewers were also obtained in full text. Full-text articles were examined for adherence to the inclusion criteria and then screened (by AC, with input from CRM and MM, in cases of uncertainty or disagreement). Grey literature were title-screened for relevance and a further full review examined adherence to inclusion criteria. 


\section{Quality assessment}

This scoping review included a non-heterogeneous sample of primary and grey literature, which made it difficult to universally apply quality assessment criteria. As standard to most scoping reviews, we did not undertake formal quality assessment and excluded papers only on grounds of relevance. As a result the analytical focus of this review centred on a critique of relevance and contribution of the included literature and did not consider methodological quality.

\section{Data extraction}

In line with Arksey and O'Malley's method, ${ }^{20}$ data extraction (charting) was multistaged. In the first stage we collected descriptive characteristics from each paper, such as study design and setting. In the second stage, findings and discussion sections of included literature were extracted into a data extraction tool (see online supplementary appendix 2) informed by NPT. ${ }^{15-17}$

The data extraction tool was designed to chart specific details of the literature and to understand factors influencing implementation. The tool was piloted on a sample $(n=5)$ of primary and grey literature, with subsequent amendments resulting in the final version. Data were extracted by independent reviewers (AC, SL, MB). Following Levac et $a l \mathrm{~s}^{21}$ recommendations, two reviewers (AC, MM) independently extracted data for a 30\% sample of primary literature to ensure approaches to extraction were consistent with each other and with the research aims.

\section{Data analysis}

A two-stage analytical process was undertaken. During the first stage, data extracted were treated as qualitative data and analysed using directed content analysis. ${ }^{25} \mathrm{~A}$ theory-informed coding framework was developed using the four main constructs and subconstructs of NPT. ${ }^{15} 16$ Data were identified and categorised to the constructs and subconstructs of NPT, exploring barriers and facilitators to implementation.

The use of NPT as a theoretical framework followed its successful application in a number of different healthcare intervention reviews. ${ }^{1326-30}$ NPT investigates and explains the successful operationalisation of interventions: how they become part of everyday practice in healthcare settings. It embodies the different types of 'work' undertaken by individuals around implementing, embedding and integrating, and allows us to understand the social structures and contexts through which new interventions are operationalised. ${ }^{14-17}$ In relation to implementation of goals of care interventions, definitions of the four core constructs and subconstructs of NPT used in this review are outlined in online supplementary appendix 3.

During the second stage, thematic analysis of the literature content was undertaken to identify, characterise and explain the factors that shape implementation of interventions that initiate communication and decision-making around goals of care. A final higher level of analytical interpretation followed, to characterise the components of contentious interventions and generate transferable learning outcomes for their implementation.

\section{RESULTS}

Searches identified 2619 citations. Following de-duplication and relevance screening, 43 sources of literature (relating to 23 interventions), including 24 items of published literature (eg, peer-reviewed papers, conference abstracts) and 19 items of grey literature (eg, conference posters, patient information documents), met the inclusion criteria and were included for data extraction (see figure 1). Table 2 provides a summary of the characteristics of included literature, the range of interventions described and the decisions of interest addressed, including DNACPR, goals of active care, supportive or palliative care, and those that are limited to communication guidelines only.

Thematic analysis of literature content suggested there were six common themes fundamental to the successful implementation of goals of care interventions: input into development, key clinical proponents, training and education, intervention workability and functionality, setting and context, and perceived value and appraisal. These are outlined below. The links between the subconstructs of NPT and these identified themes are outlined in table 3. A summary of results by NPT construct is presented in online supplementary appendix 4 as a summary and appraisal of the literature.

\section{Input into development}

The involvement of clinical staff in the development of interventions facilitated the identification of current shortfalls in practice, understanding purpose, and shaping novelty, accessibility and utility of design. ${ }^{31-35}$ Developmental input also assisted in promoting' buy-in', legitimisation and confidence in the intervention. ${ }^{31-3436-41}$ While some literature focused on the importance of input from senior, specialist individuals, ${ }^{32} 333641$ including those within implementation settings, ${ }^{33} 3442$ for intervention design, multidisciplinary collaboration in the development process was common across papers 331333436 39-45 and helpful for gaining collective knowledge from all potential users. ${ }^{31} 34363940$ In one paper, individuals who had expressed disagreement with one intervention were actively sought for involvement at each stage of development, ${ }^{31}$ highlighting how inclusion in development can contribute to overcoming barriers of individual resistance. Reconfiguration of forms and procedures was incorporated in the development and implementation process in a number of initiatives. ${ }^{31} 33343642$ The benefit of this approach was that it allowed for assessment of factors such as usability, clarity and safety, incorporating feedback into further iterations. 313342 


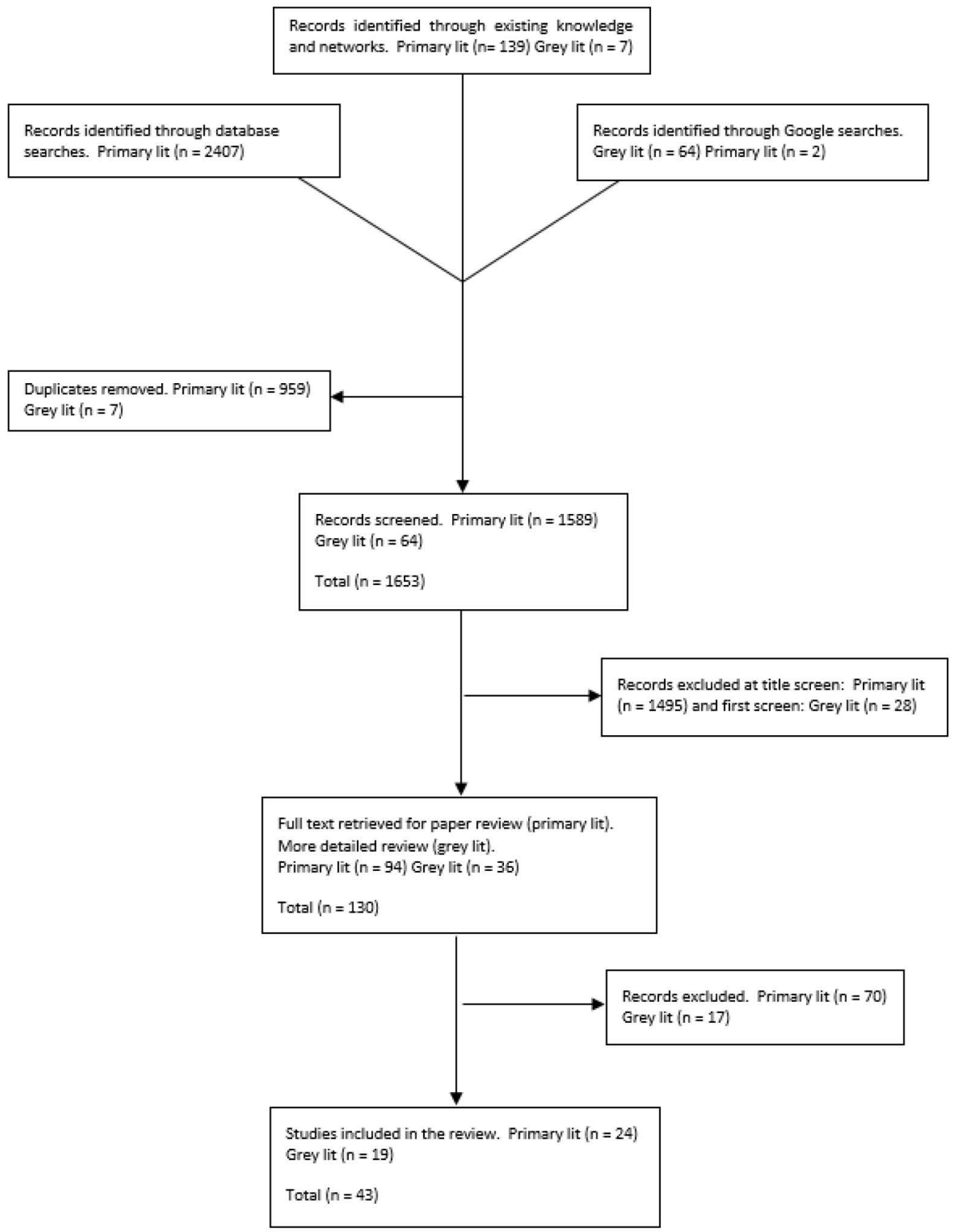

Figure 1 Flow diagram of literature screening.

\section{Key clinical proponents}

Key clinical proponents were assigned to orchestrate and lead implementation within specific sites or settings. $^{32} 424647$ They were key resources for attending and cascading training, as well as overseeing changes, embedding cultural change and providing mentorship. $^{32} 4246-48$ Their direct contact with intervention instigators appeared important in orientating and maintaining momentum of local leadership, including training and facilitation. ${ }^{32} 4247$ Similarly, support from individual senior managers in ensuring compliance with training and interventions, promoting sustained use, as well as acting as catalysts for initial implementation was valuable. $^{32} 3542464749$ Trust level managerial support was also evident through incorporation into policy and protocol $^{344549-52}$ and approval from directors. ${ }^{39} 40$ Managerial backing could be influential for organisational change, although in reality, engagement with the management of healthcare organisations was recognised by some for its complexity. ${ }^{32} 42$

\section{Training and education}

Training and education encouraged 'buyin', 3313236394246475152 promoted understanding of tasks and responsibilities, 313639424751 and facilitated a shared understanding of purpose. ${ }^{44} 41$ Staff often negatively 


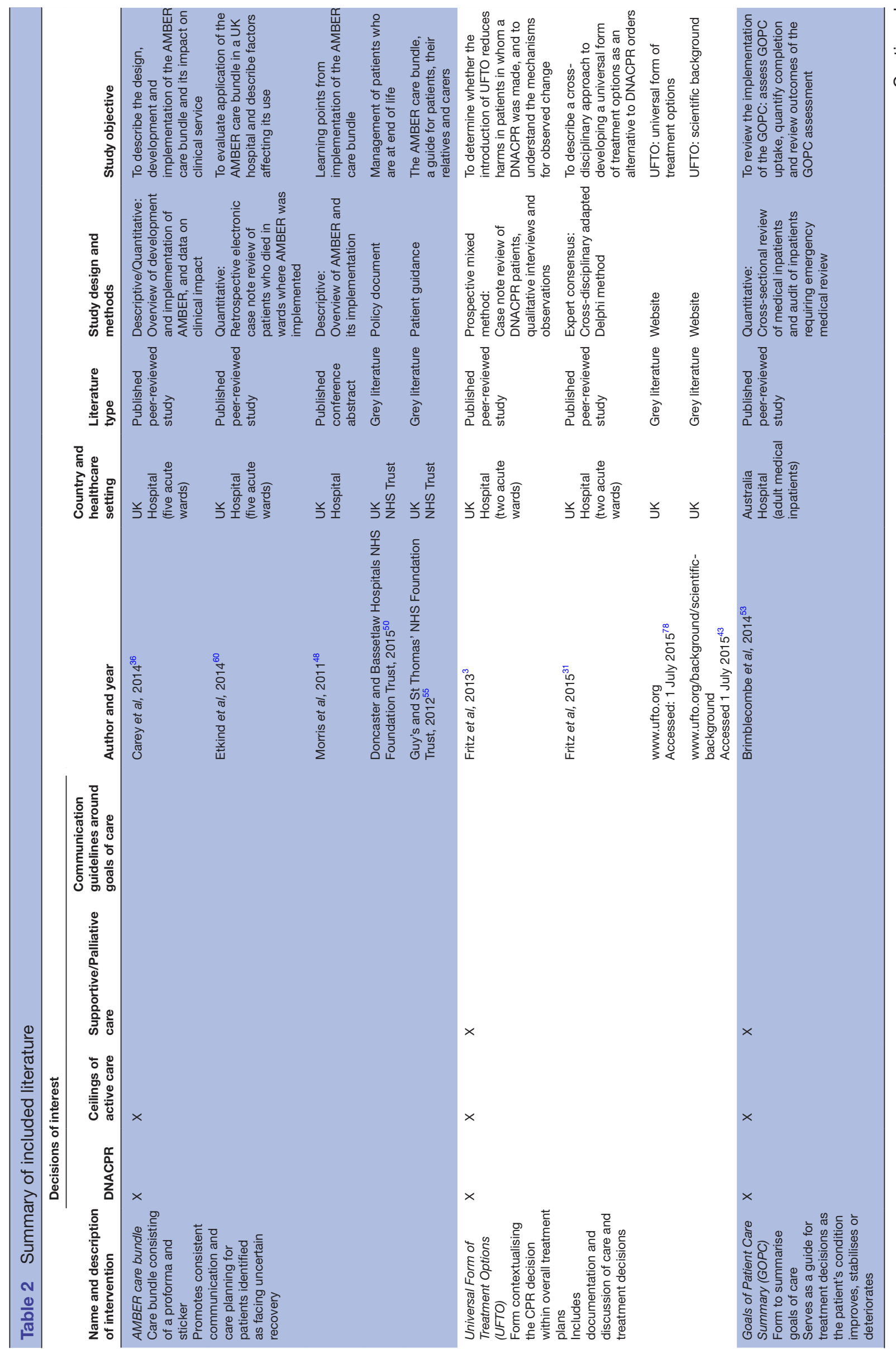




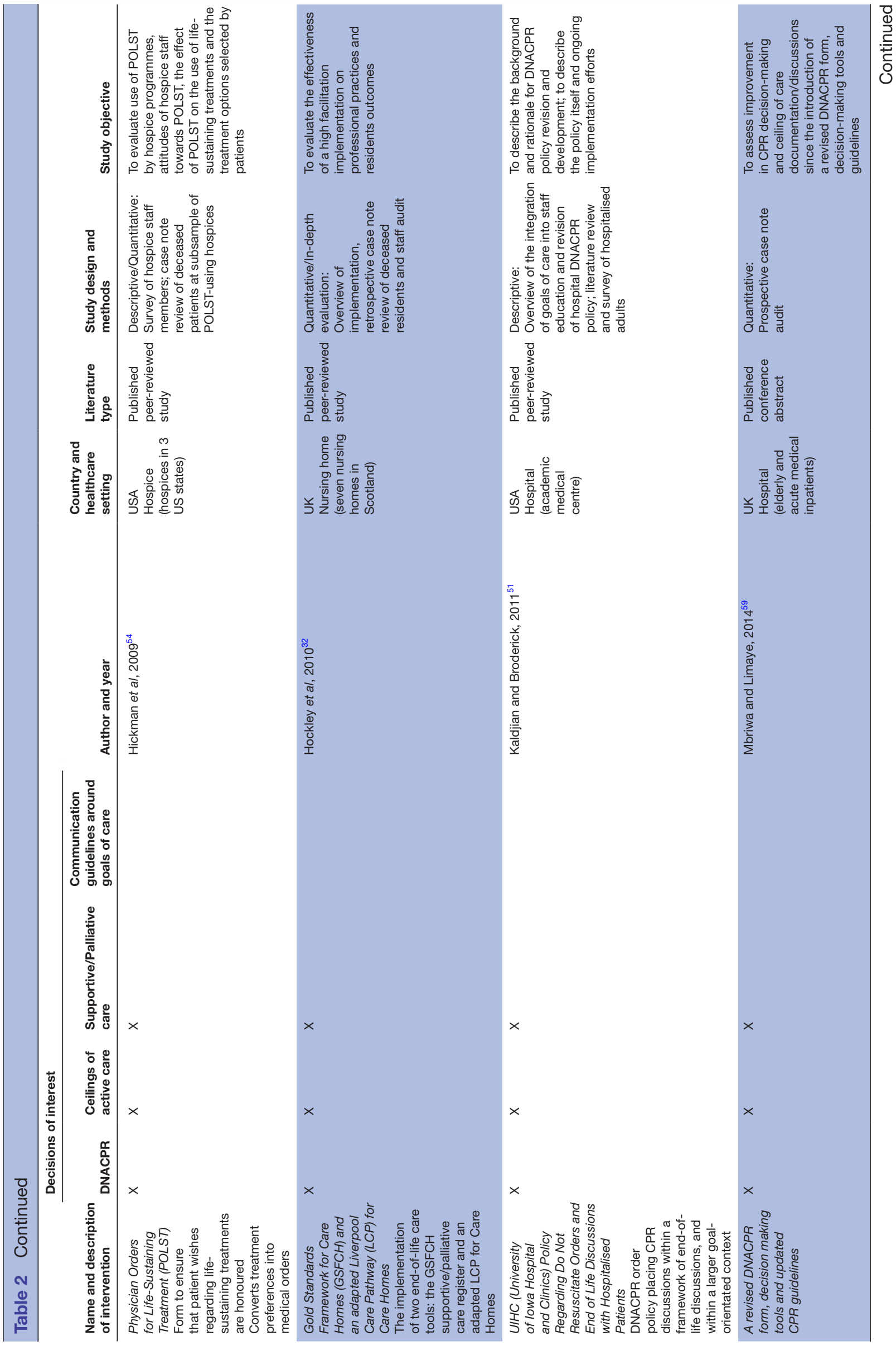




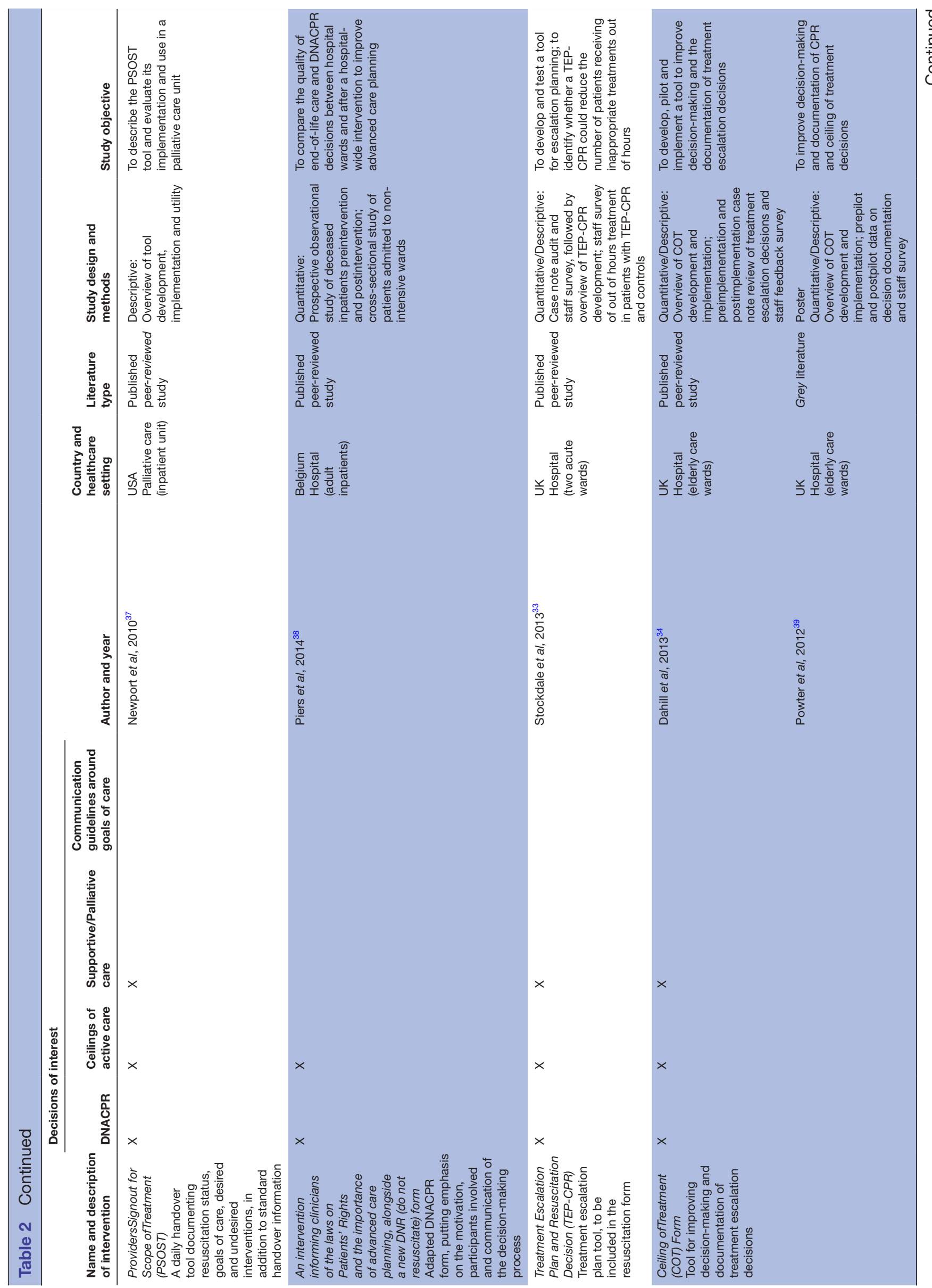




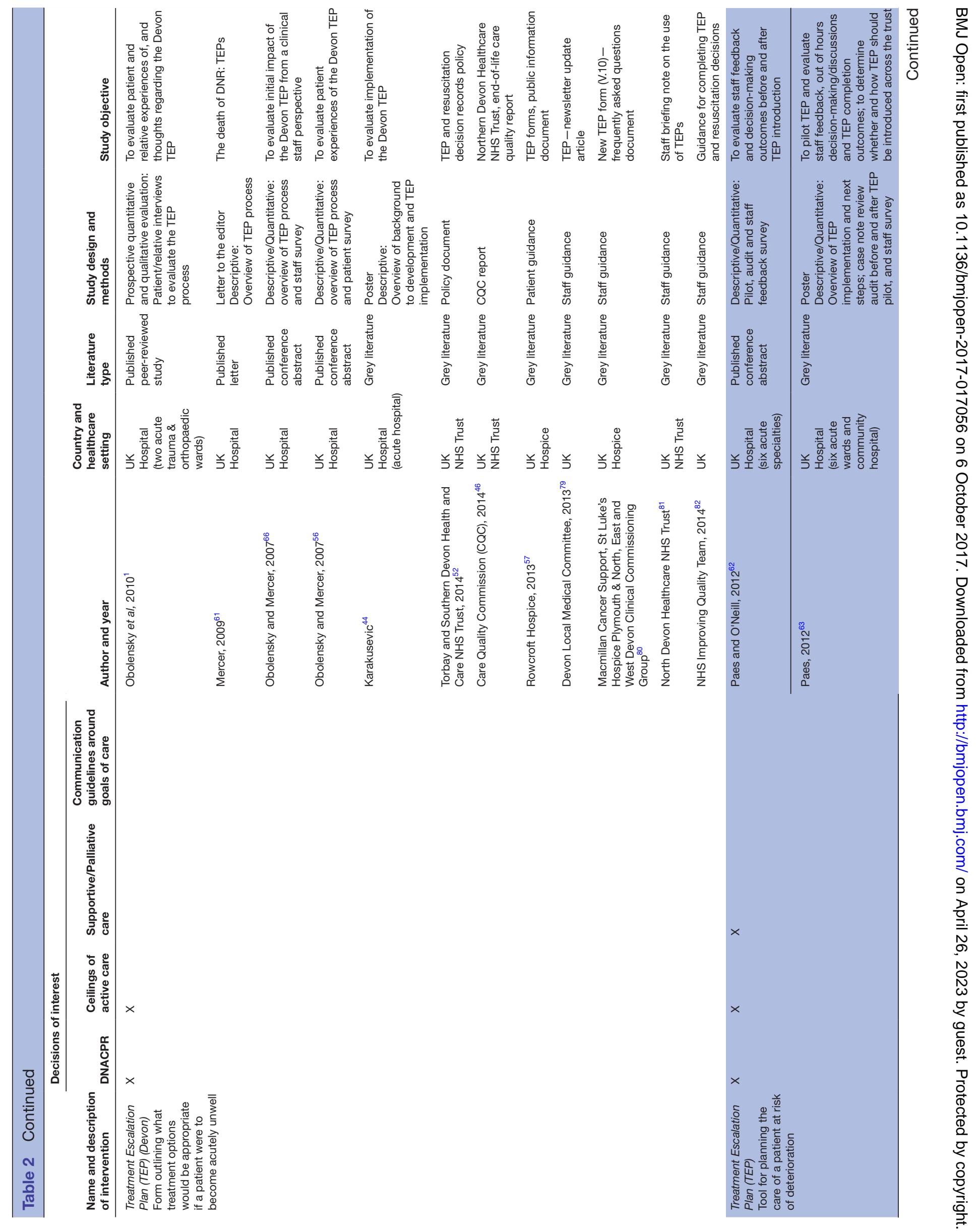




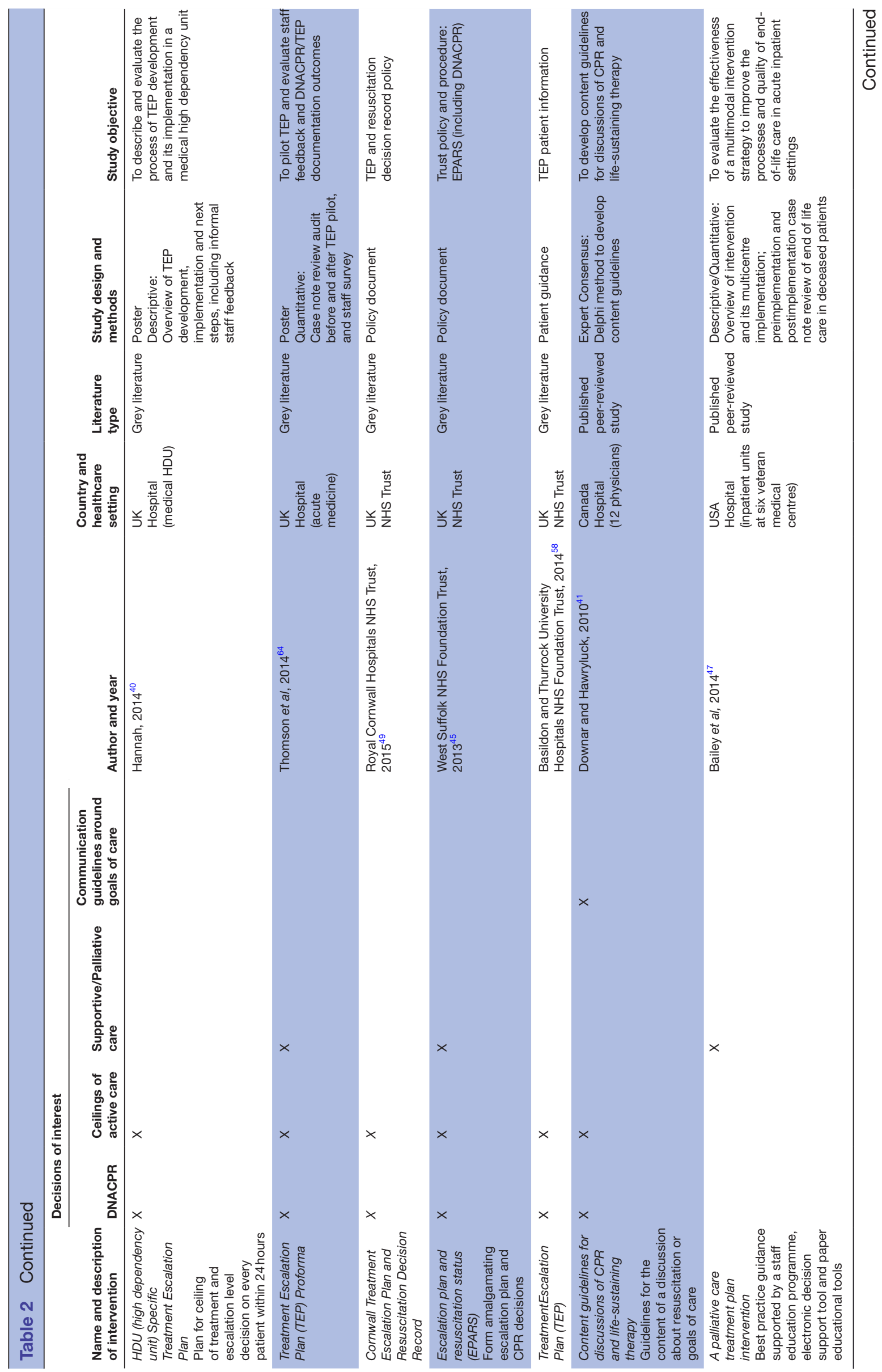

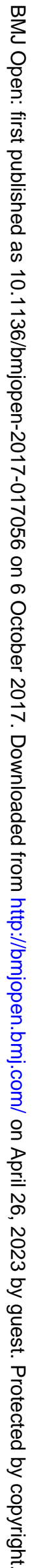




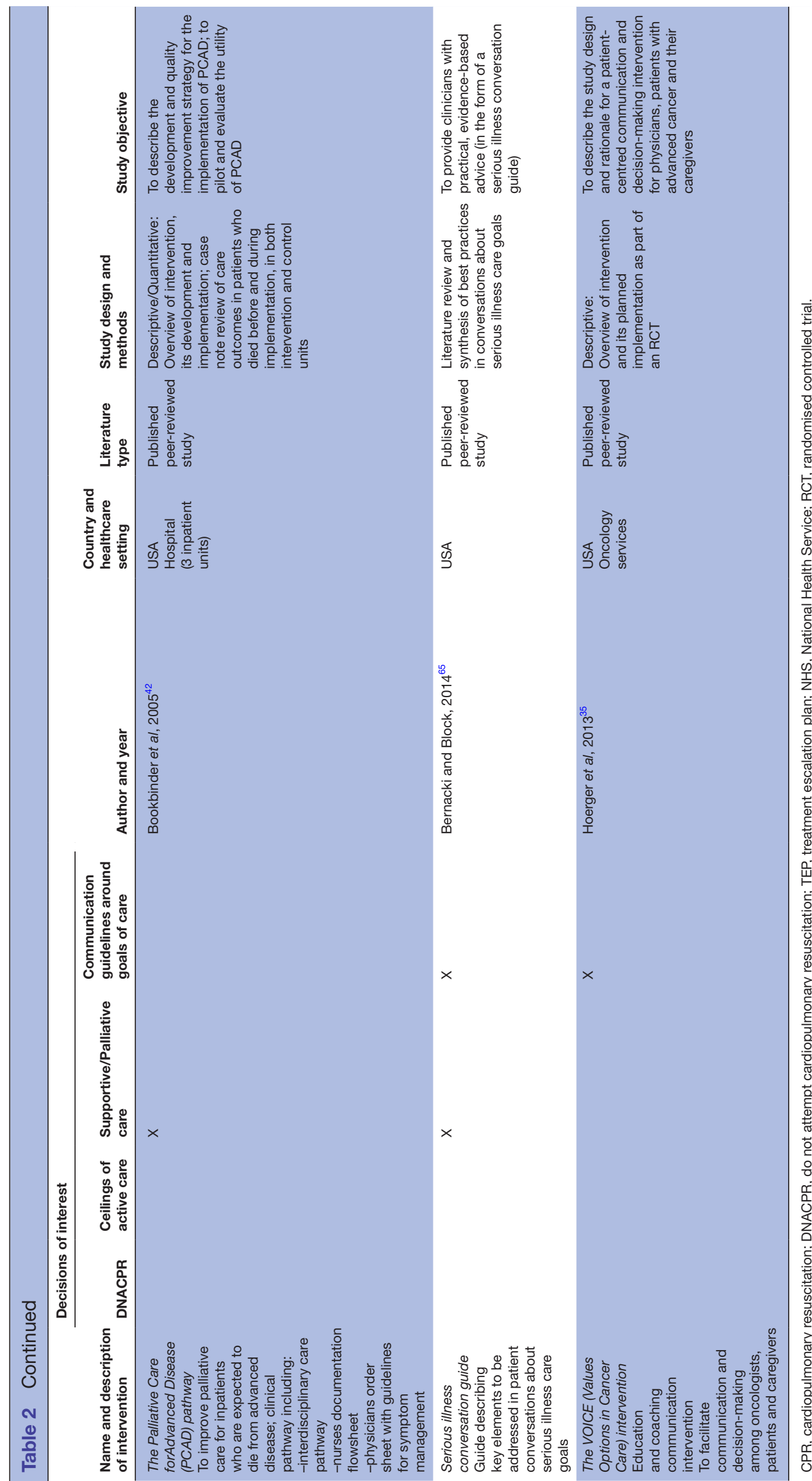

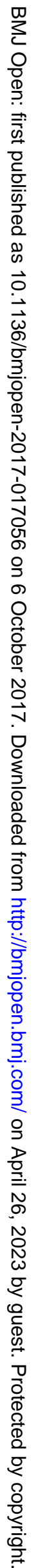




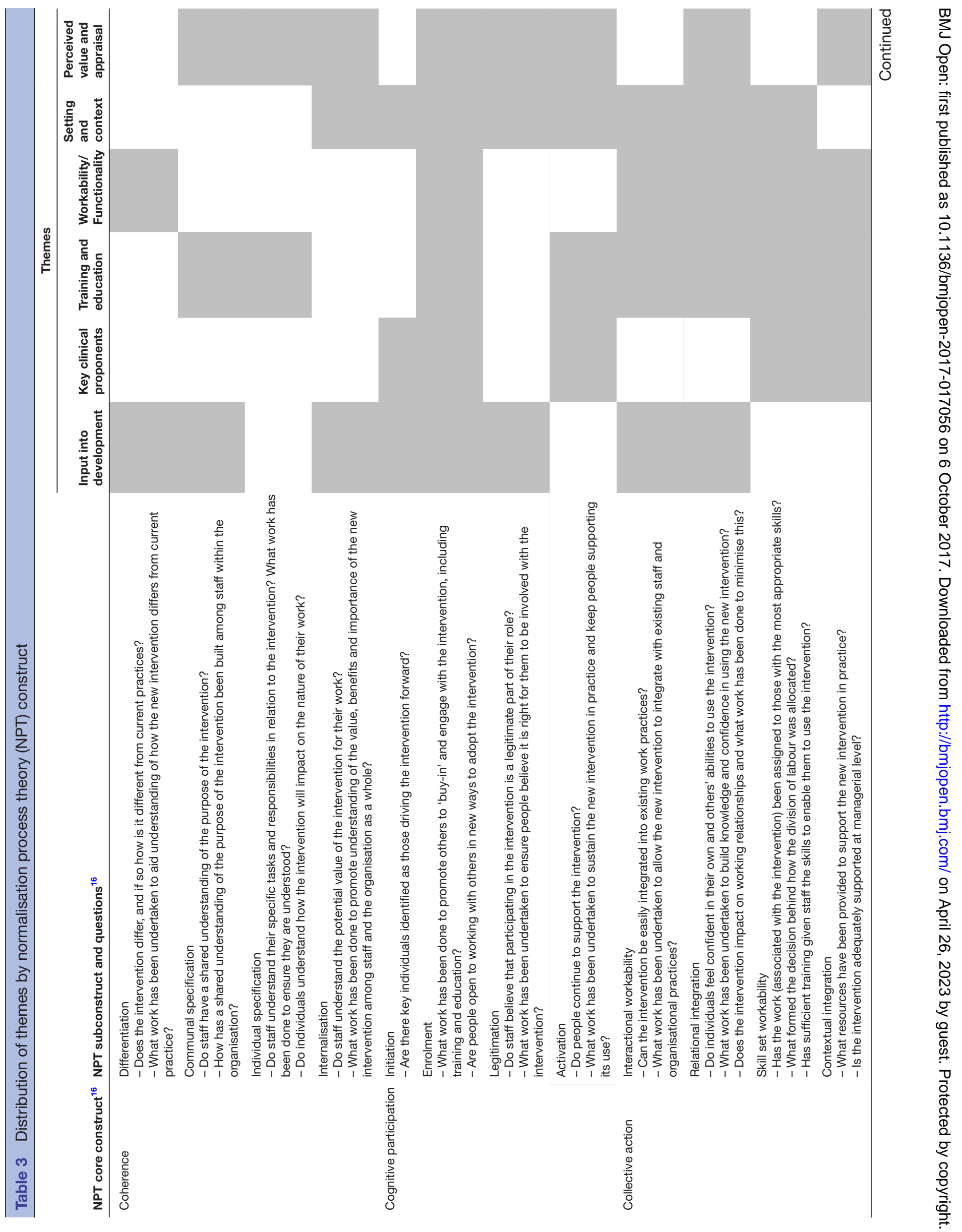


perceived the increased training requirement, ${ }^{31}$ inclusion within established working practices ${ }^{39} 424751$ and feasibility of incorporation into workload $^{35} 42$ were important considerations. Repeated sessions were used to train rotational/part-time staff, address staff turnover and enable sustained engagement. ${ }^{34} 363942$ Tailored education based on evidence and feedback facilitated the most efficacious training, especially since misunderstanding of responsibilities could result in ineffective or reduced use. ${ }^{31} 32424653$ Training in the application of skills to the intervention was fundamental, ${ }^{42} 4749$ and without this, regardless of skill level, the completion, interpretation and application of interventions in practice could be inconsistent. ${ }^{44} 4654$ Addressing communication skills was highlighted as important in a number of papers. ${ }^{35} 36384853$ Individuals responsible for training could ensure that as far as possible all staff received necessary training. ${ }^{42} 4749$ The provision of guides, algorithms and interactive materials facilitated skill development, ${ }^{35} 47$ and patient and carer information materials helped to sustain practice around newly implemented interventions. $313442454749-5255-58$

\section{Intervention workability and functionality}

A frequently adopted approach included using existing programme or frameworks (local or otherwise) as the basis for new intervention development. 32333738425459 Literature suggested healthcare practices can flex to incorporate interventions designed as part of, or alongside, existing processes. ${ }^{3} 333643474953$ This approach was often more acceptable to staff, promoting incorporation into practice, adherence and behavioural change. ${ }^{3} 334353$ Conversely, lack of transferability across healthcare settings was viewed as a barrier to implementation. ${ }^{3754}$ The workability of interventions was important, with the integral use of guidelines and prompts facilitating intervention utility and accessibility. ${ }^{13} 3134-3739-4245474951535460-65$ The use of stickers and brightly coloured forms for insertion into patient medical records promoted easy recognition of interventions. ${ }^{3654}$ For many, successful incorporation into working practices appeared to rely on the paper format of tools, their design and usability. ${ }^{13145496162}$

\section{Setting and context}

Implementation often occurred within limited clinical settings, and the direct relevance of the specialism (to the intervention) was highlighted as important for promoting its value. ${ }^{33} 3738426062-64$ Subsequent diffusion from implementation setting to hospital-wide acceptance was demonstrated in relation to a number of interventions. ${ }^{1313642}$ Despite this, multiple barriers and enablers to change were identified, including transferability across primary and secondary care settings, ${ }^{4954}$ staff turnover and management stability, ${ }^{32}$ availability of staff for updates, countersignatories to meet completion time frames, ${ }^{375354}$ time for communication processes ${ }^{33}$ robustness of implementation sites, ${ }^{32} 42$ and the difficulty in transforming clinician attitudes. ${ }^{38} 4042$ 
The clinical complexity and unpredictability surrounding the patients for whom the interventions were targeted often affected successful or opportune application. ${ }^{3360}$ Utility was improved where deterioration was predictable,${ }^{33} 3660$ yet recognition of deterioration was on occasion inherently difficult. ${ }^{60}$ Clinical uncertainty meant that at times interventions were not fully utilised for the patients they were designed to support. ${ }^{33} 36$

\section{Perceived value and appraisal}

Staff perceptions and the degree of alignment with the intended purpose of interventions were infrequently reported. ${ }^{31} 4042$ Staff valuing the intervention from the outset had important implications for effective application, ${ }^{31} 335153$ and perceived utility, existing supportive local policy and inclusive collaborative development were all highlighted as facilitators. ${ }^{31-33} 3540-434553$ At an organisational level, responsiveness to national guidance and policy was likely to promote value. ${ }^{3} 3245$ Understanding objectives early on in the implementation pathway was key, as misunderstandings were not uncommon ${ }^{36445}$ and could lead to reduced or incomplete application of interventions. $^{3653}$

Following implementation, appraisal of intervention value was frequent, ${ }^{3}{ }^{32-3439425462-6466}$ often demonstrating communal agreement centred around positive impact on working practices. ${ }^{32-34373940424854626366}$ Increased workload was expressed as a concern by staff, mainly relating to the completion of tools and the need for patient or family discussions. ${ }^{13} 37426263$ On appraisal, interventions were perceived to be a worthwhile investment due to patient benefit and the improved clarity and time saved, as a result of decisions and discussions taking place earlier in the care trajectory. ${ }^{1363} \mathrm{~A}$ number of papers recommended or proposed outcome measures relating to patient and relative experience, ${ }^{3} 34-3639404254$ but only a small number reported utilising such measures. ${ }^{1485661}$

\section{DISCUSSION}

In this review we have identified a broad and diverse literature focusing on the implementation of goals of care interventions. Findings from this review confirm these interventions are both complex and contentious in nature, and as such it is conceivable that what we have learnt here applies to other contentious interventions and processes, for example discharge planning.

Our analysis has led us to characterise the elements that constitute contentious interventions. Using the example of goals of care interventions, we propose that these interventions consist of three components that intersect at three different levels in a system of negotiated interactions:

1. negotiated decision-making between clinicians, patients and family members, which is localised and characterised by its meaning for the individual

2. the organisational procedure and collective system of making negotiated decisions
3. the sociolegal constraints of taking account of preferences (including consent and capacity) that define the parameters.

Review findings point to negotiated interaction processes taking place between individuals. This mediates a set of procedures about how decisions should be made: a set of expectations about what procedures should be done, what negotiations are possible, and what these look like within and across organisations. Sociolegal conceptualisation involves how organisations must deal with these preferences. All of these continuous components interact and affect a patient's care trajectory.

From the six themes that emerged from our analysis, we have generated transferable learning outcomes for the implementation of contentious interventions. These are described below as a series of propositions relevant to goals of care, which we contend may apply across contentious interventions as a whole. These propositions are the following:

\section{Individuals resist interventions that replicate the work of existing practices}

The value of incorporating interventions into working practices has previously been described $^{8}$ and lies in minimising disruption. The adoption of existing intervention formats and integral guidelines was popular, and may act to improve successful compliance and integration through increased staff familiarity and confidence. However there is a fine line, as individuals resist interventions that replicate the work of existing practices. ${ }^{342}$ Interventions have to be easily differentiated from other practices to be valued. There needs to be clarity regarding the practices that are discontinued, preventing unnecessary duplication, and clear identification of the benefits of the new intervention. Evidence from this review suggests that high visibility methods (eg, a sticker or coloured background) are valued for quick identification and time-saving in clinical practice. These are features unique to the paperbased nature of tools. Technological infrastructure facilitating electronic access to one record in all contexts will enhance the likelihood of widespread embedding across organisations.

\section{Contentious interventions are difficult to integrate in environments where there is clinical unpredictability and uncertainty}

Interventions aimed at improving care for patients facing uncertainty can be difficult to integrate due to the very nature of complexity that exists for these patients and their clinicians. Usually the application of skills and techniques introduces order into such situations, with clinicians seeking scripts to work to. However, clinical uncertainty can render these scripts ineffectual, leading to uncertain outcomes. In light of this, studies in this review suggest the importance of the intervention being delivered by a clinician who has an established relationship with the patient and/or knows their situation well. ${ }^{3751}$ While this represents the ideal, in the reality of clinical practice and 
a highly pressurised health service, this may prove difficult to achieve.

\section{Legitimacy is established where individuals build a shared understanding of purpose that enables them to attribute value to the intervention}

The use of senior clinicians and managers in the development process is recognised for building a shared vision. ${ }^{67}$ However, the potential for authoritative dominance of certain clinicians is recognised ${ }^{68}$ and efforts must be made to enable active contributions from key members of junior staff. A high degree of clinical ownership is recognised as important for successful implementation. ${ }^{69}$ This is more likely to be achieved if all clinicians, irrespective of seniority, believe they have a role to play and are making a valid contribution. ${ }^{70}$ Key clinical proponents are essential in building legitimacy, successful and sustained implementation, especially in the context of organisational instability. ${ }^{71} 72$

\section{Training and education provide the framework for individuals to understand the value of what they do}

Training and education have an important role in facilitating a shared understanding of purpose, addressing both moral and technical aspects, which are important for building value in the intervention. Training and education serve to facilitate this, fostering clarity of value in the intervention.

The importance of training and education is not a new concept. However, there is a lack of agreement regarding optimal delivery methods, which may relate to a need for training to fit within the clinical context. Findings from the review point to the need for multimodal educational programmes, repetition and timeliness of training, but this should be considered in the context of staff and financial resource implications. ${ }^{73} 74$

\section{Appraisal work is critically important because it is how individuals value the intervention}

The importance of sharing appraisal outcomes, conveying purpose and value to others is evident, as value is often not realised until successful implementation is achieved. However, reporting of this in the literature was limited ${ }^{346}$ In particular, evaluation of patient and family perspectives was notably absent in the papers included in this review. We suggest their inclusion is crucial for imputing value into interventions that seek to include them. ${ }^{75}$ Surveys suggest that clinicians will foresee advantages and disadvantages in any proposed intervention. ${ }^{3133}$ Therefore, sharing appraisal work, which addresses the positives and negatives, may be beneficial in providing a more balanced view of an intervention's value.

\section{The transfer of contentious interventions to other settings is problematic}

The initial setting can influence implementation success and subsequent transfer to other locations. In our review there is limited evidence of transfer at scale, over multiple geographical sites. ${ }^{47}$ There are multiple barriers through which interventions need to filter, including specialisms, care locations, and structural and cultural factors. In addition, groups of clinicians hold different values, skills and knowledge. This introduces problems of discretion, interpretation and enactment within different contexts and may limit scale-up and transferability. While not widely addressed by the papers in this review, wider implementation literature suggests the importance of involving patients and caregivers when transferring and implementing interventions beyond formal clinical settings. ${ }^{76} 77$

In summary, improving decision-making around goals of care and ensuring patient preferences are taken into account require a better understanding of the implementation processes and factors that promote or impede implementation. We have used the exemplar of goals of care interventions to elucidate propositions in relation to the successful implementation of contentious interventions, where success refers to the routine incorporation and embedding of intervention components into everyday practice. We believe these propositions to be transferable and generalisable beyond the remit of goals of care, to other contentious (and complex) healthcare interventions.

\section{CONCLUSION}

Findings from the review show that while such interventions are variable in design and use, there are a series of collective factors that influence successful implementation into routine clinical practice. We recommend that those seeking to introduce goals of care and other contentious interventions consider the different facets of NPT and use knowledge of this to develop implementation strategies. The contentious nature of these interventions means that their incorporation into everyday practice is dependent on a number of factors. Building a shared understanding of purpose that enables participants to attribute value to the intervention is key, and both training and education and appraisal work, play an important role in this process. Identifying clinical proponents who are able to, not only drive, but positively influence implementation is essential. Implementing complex and contentious interventions presents challenges that operate at an individual, organisational and systems level. It is these interaction level processes that make an intervention contentious, as well as making it challenging to introduce and embed. Success is more likely to occur, be established and sustained, if due attention is paid to the processes that facilitate operationalisation in the healthcare settings in which implementation occurs.

Acknowledgements This review was funded by the National Institute for Health Research (NIHR) Collaboration for Leadership in Applied Health Research and Care Wessex. The views expressed are those of the authors and not necessarily those of the NHS, the NIHR or Department of Health. The authors would like to thank Dr Mark Banting for his assistance with data extraction in the initial phase of the review.

Contributors MM, CRM and AR designed the review. AC performed searches. AC and CRM screened titles and abstracts, and full paper screening was carried out 
by AC, MM and CRM. Data extraction was performed by AC, MM and SL. AC and MM drafted the manuscript with assistance from SL, CRM, NC and AR. All authors critically reviewed the manuscript for intellectual content and approved the final version of the paper. $\mathrm{AC}$ is the guarantor.

Funding This work was supported by the National Institute for Health Research Collaboration for Leadership in Applied Health Research and Care (NIHR CLAHRC) Wessex which is a partnership between Wessex NHSorganisations and partners and the University of Southampton. Funders had no role in study design, data collection and analysis, decision to publish or preparation of the manuscript.

Disclaimer The views expressed are those of the author(s) and not necessarily those of the NHS, the NIHR or the Department of Health.

Competing interests None declared.

Provenance and peer review Not commissioned; externally peer reviewed.

Data sharing statement № additional data are available.

Open Access This is an Open Access article distributed in accordance with the terms of the Creative Commons Attribution (CC BY 4.0) license, which permits others to distribute, remix, adapt and build upon this work, for commercial use, provided the original work is properly cited. See: http://creativecommons.org/ licenses/by/4.0/

(c) Article author(s) (or their employer(s) unless otherwise stated in the text of the article) 2017. All rights reserved. No commercial use is permitted unless otherwise expressly granted.

\section{REFERENCES}

1. Obolensky L, Clark T, Matthew G, et al. A patient and relative centred evaluation of treatment escalation plans: a replacement for the donot-resuscitate process. J Med Ethics 2010;36:518-20.

2. Findlay GP, Shotton H, Kelly K, et al. Time to intervene? A review of patients who underwent cardiopulmonary resuscitation as a result of an in-hospital cardiorespiratory arrest.A report by the National Confidential Enquiry into Patient Outcome and Death. 2012.

3. Fritz Z, Malyon A, Frankau JM, et al. The Universal Form of Treatment Options (UFTO) as an alternative to Do Not Attempt Cardiopulmonary Resuscitation (DNACPR) orders: a mixed methods evaluation of the effects on clinical practice and patient care. PLoS One 2013;8:e70977.

4. Nicholson TR, Cutter W, Hotopf M. Assessing mental capacity: the Mental Capacity Act. BMJ 2008;336:322-5.

5. Craig P, Dieppe P, Macintyre S, et al. Developing and evaluating complex interventions: the new Medical Research Council guidance. BMJ 2008;337:a1655.

6. Council of Europe (1950) European Convention for the Protection of Human Rights and Fundamental Freedoms, as amended by Protocols Nos. 11 and 14, November 4 (ets, no. 5). http://www. refworld.org/docid/3ae6b3b04.html (accessed 08 Mar 2017).

7. May C, Finch T, Mair F, et al. Understanding the implementation of complex interventions in health care: the normalization process model. BMC Health Serv Res 2007;7:148.

8. May C. Agency and implementation: understanding the embedding of healthcare innovations in practice. Soc Sci Med 2013;78:26-33.

9. Damschroder LJ, Aron DC, Keith RE, et al. Fostering implementation of health services research findings into practice: a consolidated framework for advancing implementation science. Implement Sci 2009;4:1.

10. Moore GF, Audrey S, Barker M, et al. Process evaluation of complex interventions: Medical Research Council guidance. BMJ 2015;350:h1258.

11. May CR, Johnson M. Implimentation context and complexity. Implement Sci 2016;11:141.

12. May C. Mobilising modern facts: health technology assessment and the politics of evidence. Sociol Health IIIn 2006;28:513-32.

13. Lund S, Richardson A, May C. Barriers to advance care planning at the end of life: an explanatory systematic review of implementation studies. PLoS One 2015;10:e0116629.

14. May C, Finch T. Implementing, Embedding, and Integrating Practices: An Outline of Normalization Process Theory. Sociology 2009;43:535-54.

15. May CR, Mair F, Finch T, et al. Development of a theory of implementation and integration: Normalization Process Theory. Imple Sci 2009;4.1.

16. May C, Rapley T, Mair FS, et al. Normalization Process Theory On-line Users' Manual, Toolkit and NoMAD instrument, 2015. http:// www.normalizationprocess.org
17. Finch TL, Girling M, May CR, et al. NoMAD: Implementation measure based on Normalization Process Theory [Measurement instrument]. 2015. http://www.normalizationprocess.org

18. Pham MT, Rajić A, Greig JD, et al. A scoping review of scoping reviews: advancing the approach and enhancing the consistency. Res Synth Methods 2014;5:371-85.

19. Davis K, Drey N, Gould D. What are scoping studies? A review of the nursing literature. Int J Nurs Stud 2009;46:1386-400.

20. Arksey H, O'Malley L. Scoping studies: towards a methodological framework. Int J Soc Res Methodol 2005;8:19-32.

21. Levac D, Colquhoun H, O'Brien KK. Scoping studies: advancing the methodology. Implement Sci 2010;5:1.

22. Fritz Z, Cork N, Dodd A, et al. DNACPR decisions: challenging and changing practice in the wake of the Tracey judgment. Clin Med 2014;14:571-6.

23. Perkins GD, Griffiths F, Slowther A-M, et al. Do-not-attemptcardiopulmonary-resuscitation decisions: an evidence synthesis. HSDR 2016;4:1-154.

24. RESPECT: Recommended Summary Plan for Emergency Care and Treatment (2017). www.respectprocess.org.uk (accessed $05 \mathrm{Jul}$ 2017).

25. Hsieh HF, Shannon SE. Three approaches to qualitative content analysis. Qual Health Res 2005;15:1277-88.

26. Watson R, Parr JR, Joyce C, et al. Models of transitional care for young people with complex health needs: a scoping review. Child Care Health Dev 2011;37:780-91.

27. Johnson MJ, May CR. Promoting professional behaviour change in healthcare: what interventions work, and why? A theory-led overview of systematic reviews. BMJ Open 2015;5:e008592.

28. Gallacher K, Morrison D, Jani B, et al. Uncovering treatment burden as a key concept for stroke care: a systematic review of qualitative research. PLoS Med 2013;10:e1001473.

29. Mair FS, May C, O'Donnell C, et al. Factors that promote or inhibit the implementation of e-health systems: an explanatory systematic review. Bull World Health Organ 2012;90:357-64.

30. May C, Sibley A, Hunt K. The nursing work of hospital-based clinical practice guideline implementation: an explanatory systematic review using Normalisation Process Theory. Int J Nurs Stud 2014;51:289-99.

31. Fritz Z, Fuld JP. Development of the Universal Form Of Treatment Options (UFTO) as an alternative to Do Not Attempt Cardiopulmonary Resuscitation (DNACPR) orders: a cross-disciplinary approach. J Eval Clin Pract 2015;21:109-17.

32. Hockley J, Watson J, Oxenham D, et al. The integrated implementation of two end-of-life care tools in nursing care homes in the UK: an in-depth evaluation. Palliat Med 2010;24:828-38.

33. Stockdale C, Trivedi B, Jerome E, et al. Implementation of a combined Cardiopulmonary Resuscitation and Treatment Escalation Plan document in a District General Hospital. BMJ Qual Improv Rep 2014;2:u202653.w1236.

34. Dahill M, Powter L, Garland L, et al. Improving documentation of treatment escalation decisions in acute care. BMJ Qual Improv Rep 2013;2:u200617.w1077.

35. Hoerger M, Epstein RM, Winters PC, et al. Values and options in cancer care (VOICE): study design and rationale for a patientcentered communication and decision-making intervention for physicians, patients with advanced cancer, and their caregivers. BMC Cancer 2013;13:188.

36. Etkind SN, Karno J, Edmonds PM, et al. Supporting patients with uncertain recovery: the use of the AMBER care bundle in an acute hospital. BMJ Support Palliat Care 2015;5:95-8.

37. Newport KB, Patel S, Lyckholm L, et al. The "PSOST": Providers Signout for Scope of Treatment. J Palliat Med 2010;13:1055-8.

38. Piers RD, Benoit DD, Schrauwen WJ, et al. Do-not-resuscitate decisions in a large tertiary hospital: differences between wards and results of a hospital-wide intervention. Acta Clinica Belgica 2014;66:2:116-22.

39. Powter L, Dahill M, Garland L, et al. Improving decision making and documentation of resuscitation and ceiling of treatment decisions. http://www.acutemedicine.org.uk/wp-content/uploads/ 2012/10/aqi28\%20-\%20improving\%20decision\%20making\% 20and\%20documentation\%20of\%20resuscitation\%20and\% 20ceiling\%20of\%20treatment\%20decisions.pdf (accessed 27 June 2016).

40. Hannah J. One TEP at a time treatment escalation plans in medical high dependency. 2014. http://www.qihub.scot.nhs.uk/media/ 618301/julie\%20hannah.pdf (accessed 08 Marc 2017).

41. Downar J, Hawryluck L. What should we say when discussing "code status" and life support with a patient? A Delphi analysis. J Palliat Med 2010;13:185-95. 
42. Bookbinder M, Blank AE, Arney E, et al. Improving end-of-life care: development and pilot-test of a clinical pathway. J Pain Symptom Manage 2005;29:529-43.

43. Universal Form of Treatment Options: Scientific Background (2015). http://www.ufto.org/background/scientific-background/ (accessed 01 Jul 2015).

44. Karakusevic C. Devon Treatment Escalation Plan \& Resuscitation Decision Record (TEP). http://www.macmillan.org.uk/documents/ aboutus/health_professionals/ primarycare/showcasing/2013/ treatment-escalation-and-resuscitation-devon.pdf (accessed 23 Jun 2016).

45. West Suffolk NHS Foundation Trust Resuscitation Committee. Escalation Plan and Resuscitation Status (EPARS) (including DNACPR).West Suffolk NHS Foundation Trust. 2013.

46. Care Quality Commission. North Devon Healthcare NHS Trust End of Life Care Quality Report. End of Life Care Quality Report. 2014.

47. Bailey FA, Williams BR, Woodby LL, et al. Intervention to improve care at life's end in inpatient settings: the BEACON trial. $J$ Gen Intern Med 2014;29:836-43.

48. Morris M, Briant L, Chidgey-Clark J, et al. Bringing in care planning conversations for patients whose recovery is uncertain: learning from the AMBER care bundle. BMJ Support Palliat Care 2011;1:72.

49. Over J. Treatment Escalation Plan and Resuscitation Decision Record (in relation to the adult patient over 18 years). Royal Cornwall Hospitals NHS Trust. 2015.

50. Nutt S, Lanaghan K. Management of Patients who are End of Life. Doncaster and Bassetlaw Hospitals NHS Foundation Trust. 2015.

51. Kaldjian LC, Broderick A. Developing a policy for do not resuscitate orders within a framework of goals of care. Jt Comm J Qual Patient Saf 2011;37:11-19.

52. Gray C, Plans TE. Treatment Escalation Plans (TEP) and Resuscitation Decision Records. Torbay and Southern Devon Health and Care NHS Trust. 2014.

53. Brimblecombe C, Crosbie D, Lim WK, et al. The Goals of Patient Care project: implementing a proactive approach to patient-centred decision-making. Intern Med J 2014;44:961-6.

54. Hickman SE, Nelson CA, Moss AH, et al. Use of the Physician Orders for Life-Sustaining Treatment (POLST) paradigm program in the hospice setting. J Palliat Med 2009;12:133-41.

55. Guy's and St Thomas' NHS Foundation Trust. The AMBER care bundle - a guide for patients, their relatives and carers. Patient information leaflet 2012.

56. Obolensky L, Mercer M. Patients' Experiences of Treatment Escalation Plans The Intensive Care Society Annual Spring Meeting. 2007.

57. Human S, Scott K. Treatment Escalation Plan (TEP) Forms. Rowcroft Hospice 2013.

58. Basildon and Thurrock University Hospitals NHS Foundation Trust Critical care. Treatment Escalation Plan (TEP) Basildon and Thurrock University Hospitals NHS Foundation Trust. 2014

59. Mbriwa K, Limaye S. 44 * HOW CAN WE IMPROVE 'DO NOT ATTEMPT CARDIOPULMONARY RESUSCITATION' (DNACPR) AND END OF LIFE CARE (EOLC) DECISION MAKING? Age Ageing 2014;43:10.

60. Etkind SN, Karno J, Edmonds PM, et al. Supporting patients with uncertain recovery: the use of the AMBER care bundle in an acute hospital. BMJ Support Palliat Care 2015;5:95-8.

61. Mercer MH. The death of DNR: Treatment escalation plans. (Letter to the editor). BMJ 2009;338:b2020.

62. Paes P, O'Neill C. Treatment escalation plans - a tool to aid end of life decision making? BMJ Support Palliat Care 2012;2(Supp 1):A60.1-A60.
63. Paes P. Treatment Escalation Planning - Introducing systematic senior clinical decision making. http://www.karenpicking.co.uk/ images/Paul\%20Paes\%20-\%20project\%20poster.pdf (accessed: 23 June 2016)

64. Thomson B, Kok K, Harrod E, et al. Introducing Treatment Escalation Plans in Acute Medicine. http://www.acutemedicine.org.uk/wpcontent/uploads/2014/10/AQI-40-Introducing-Treatment-EscalationPlans-in-Acute-Medicine-A-Quality-Improvement-Project.pdf (accessed 23 Jun 2016).

65. Bernacki RE, Block SD. American College of Physicians High Value Care Task Force. Communication about serious illness care goals: a review and synthesis of best practices. JAMA Intern Med 2014;174:1994-2003.

66. Obolensky L, Mercer M. Replacing Do-Not-Resuscitate Forms with Treatment Escalation Plans. The Intensive Care Society Annual Spring Meeting. 2007.

67. Markóczy L. Consensus formation during strategic change. SMJ 2001;22:1013-31.

68. Nugus P, Greenfield D, Travaglia J, et al. How and where clinicians exercise power: interprofessional relations in health care. Soc Sci Med 2010;71:898-909.

69. Yana R, Jo RM. Getting guidelines into practice: a literature review. Nurs Stand 2004:18:33-40.

70. Checkland PB. Systems thinking, systems practice. Chichester: John Wiley, 1981.

71. Stetler CB, Legro MW, Rycroft-Malone J, et al. Role of "external facilitation" in implementation of research findings: a qualitative evaluation of facilitation experiences in the Veterans Health Administration. Implement Sci 2006;1:1.

72. Locock L, Dopson S, Chambers D, et al. Understanding the role of opinion leaders in improving clinical effectiveness. Soc Sci Med 2001;53:745-57.

73. Phillipson L, Goodenough B, Reis S, et al. Applying Knowledge Translation Concepts and Strategies in Dementia Care Education for Health Professionals: Recommendations From a Narrative Literature Review. J Contin Educ Health Prof 2016;36:74-81.

74. Field RA, Fritz Z, Baker A, et al. Systematic review of interventions to improve appropriate use and outcomes associated with do-notattempt-cardiopulmonary-resuscitation decisions. Resuscitation 2014;85:1418-31.

75. National Institute for Health Research. Patients and the public 2016. http://www.nihr.ac.uk/patients-and-public/ (accessed 04 Nov 2016).

76. May C, Montori VM, Mair FS. We need minimally disruptive medicine. BMJ 2009;339:b2803.

77. Shippee ND, Shah ND, May CR, et al. Cumulative complexity: a functional, patient-centered model of patient complexity can improve research and practice. J Clin Epidemiol 2012;65:1041-51.

78. UFTO: Universal Form of Treatment Options. 2015. www.ufto.org (accessed 01 Jul 2015).

79. Devon LMC Newsletter. Treatment Escalation Plans (TEP) - new update. Newsletter article. 2013.

80. Hill S, Mitchell P, Cooper J, et al. New TEP form (Version 10) Frequently Asked Questions Macmillan Cancer Support, St Luke's Hospice Plymouth, East and West Devon Clinical Commissioning Group.

81. Diamond A. Briefing note on the use of Treatment Escalation Plans (TEPs) North Devon Healthcare NHS Trust.

82. North Devon Healthcare NHS Trust. Guidance for completing Treatment Escalation Plan and Resuscitation Decisions. http:// www.northdevonhealth.nhs.uk/wp-content/uploads /2015/07/V10FINAL-Guidance-for-TEP-and-Flowchart.pdf (accessed 21 Mar 2017). 